\title{
Near-Earth Object Characterization \\ Using Ground-Based Radar Systems
}

A White Paper Submitted to the

Planetary Science and Astrobiology Decadal Survey 2023-2032

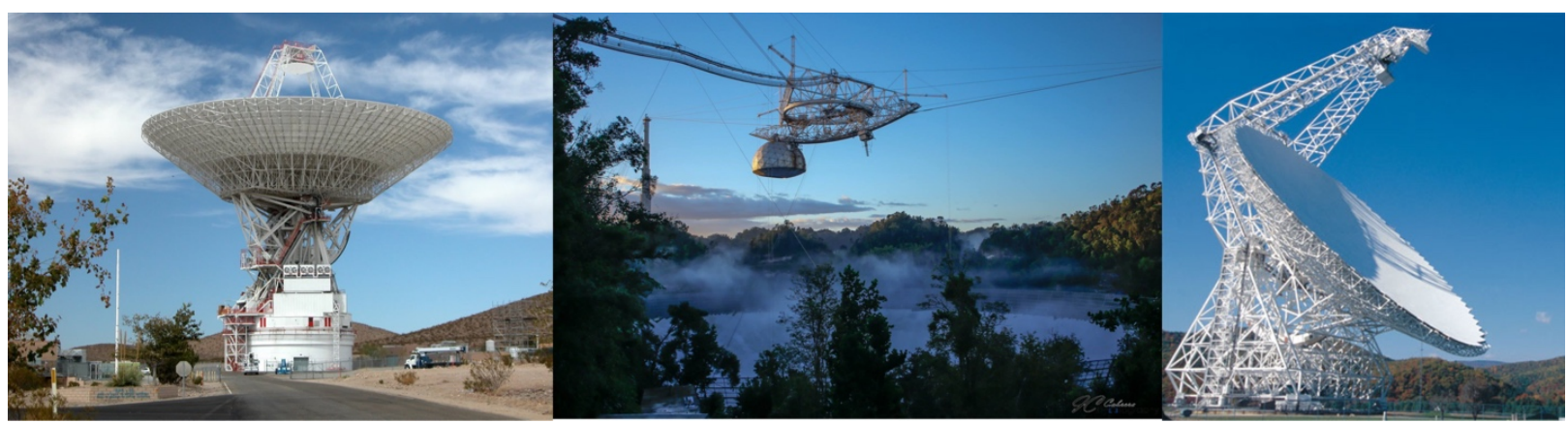

Image credit (from the left): Hal Janzen, Israel Cabrera, and National Radio Astronomy Observatory

Anne K. Virkki ${ }^{1}$ (Contact: avirkki@naic.edu, +1 (787) 878-2612 ext. 337)

Co-authors: Patrick A. Taylor ${ }^{2}$, Michael W. Busch ${ }^{3}$, Ellen S. Howell ${ }^{4}$, Edgard G. Rivera-Valentín ${ }^{2}$, Yanga Fernandez ${ }^{5}$, Lance A.M. Benner ${ }^{6}$, Marina Brozović ${ }^{6}$, Tracy M. Becker ${ }^{7}$, Sean E. Marshall ${ }^{1}$, Dylan C. Hickson ${ }^{1}$, Joseph Masiero ${ }^{6}$, Alessondra Springmann ${ }^{4}$, Flora Paganelli ${ }^{3}$, Flaviane C.F. Venditti ${ }^{1}$, Luisa F. Zambrano Marín ${ }^{1}$, Andy Lopez Oquendo ${ }^{8}$, Maria Womack ${ }^{9}$, Melissa J. Brucker $^{4}$, Bruce A. Campbell ${ }^{10}$, Michael C. Nolan ${ }^{4}$

Endorsers: Paul A. Abell ${ }^{11}$, Betzaida Aponte Hernandez ${ }^{2}$, Amber Bonsall ${ }^{12}$, Maxime Devogèle ${ }^{1}$, Frank D. Ghigo $^{12}$, Amara Graps ${ }^{13}$, Alain Herique ${ }^{14}$, Devanshu Jha ${ }^{15}$, Christopher Magri ${ }^{16}$, Amy K. Mainzer ${ }^{4}$, Jean-Luc Margot $^{17}$, Shantanu P. Naidu ${ }^{6}$, G. Wesley Patterson ${ }^{18}$, Noemí Pinilla-Alonso ${ }^{9}$

${ }^{1}$ Arecibo Observatory (University of Central Florida), PR, USA

${ }^{2}$ Lunar and Planetary Institute, Universities Space Research Association, TX, USA

${ }^{3}$ SETI Institute, CA, USA

${ }^{4}$ University of Arizona, AZ, USA

${ }^{5}$ University of Central Florida, FL, USA

${ }^{6}$ Jet Propulsion Laboratory, California Institute of Technology, CA, USA

${ }^{7}$ Southwest Research Institute, TX, USA

${ }^{8}$ Northern Arizona University, AZ, USA
${ }^{9}$ Florida Space Institute (University of Central Florida), FL, USA

${ }^{10}$ Smithsonian Institution, DC, USA

${ }^{11}$ NASA Johnson Space Center, TX, USA

${ }^{12}$ Green Bank Observatory, WV, USA

${ }^{13}$ Planetary Science Institute, AZ, USA

${ }^{14}$ University of Grenoble, France

${ }^{15} \mathrm{MVJ}$ College of Engineering, India

${ }^{16}$ University of Maine at Farmington, MA, USA

${ }^{17}$ University of California, Los Angeles, CA, USA

${ }^{18}$ Johns Hopkins Applied Physics Laboratory, MD, USA 


\section{Executive Summary}

Ground-based planetary radar systems are a crucial tool for post-discovery high-precision astrometry and physical characterization of near-Earth asteroids and comets. Radar ranging and imaging capabilities exceed those of any other ground-based instruments. As described in more detail in the Decadal white paper (henceforth WP) Mainzer et al. and WP Taylor et al., detection and characterization of potentially hazardous asteroids was mandated by the US Congress through George E. Brown, Jr. Near-Earth Object Survey Act in 2005. While optical telescopes survey for new asteroid detections, post-discovery characterization using planetary radar can secure the heliocentric orbit information, extending the interval of reliable orbit estimation by decades to centuries for most objects and preventing recently-discovered objects from being lost. Radar observations also provide constraints on the object's size, rotation state, morphology, satellites, and near-surface geology as well as regolith properties, making groundbased radar systems a powerful, low-cost complement to spacecraft destined for specific targets. Furthermore, they can be used for characterizing the nuclei of comets, which are typically obscured by the dust coma at optical wavelengths, and probe the decimeter-scale coma particle abundance, which is relevant especially for the study of disintegrating comets. These characteristics are invaluable information for understanding the formation and evolution of asteroids and comets, which are the building blocks of our Solar System, for evaluating impact risk and developing impact mitigation technologies, and for ensuring safe spacecraft encounters. In the next decade, in addition to continuing as a state-of-the-art astrometric tool for planetary defense, we expect ground-based radar observations to expand our knowledge of the physical characteristics of the near-Earth asteroid population in terms of their shape, binarity, and near-surface structure. We recommend that facilities with ground-based radar systems, planetary radar programs, and research and analysis of radar observations receive full support from NASA and NSF to ensure their availability to the planetary science community and as a resource for planetary defense initiatives through the next decade.

\section{Introduction}

Ground-based planetary radar systems have been used for characterization of small bodies since 1968 [1]. The Arecibo Observatory (AO) and Goldstone Solar System Radar (GSSR) in Puerto Rico and California, respectively, have observed to date more than 950 individual nearEarth asteroids (NEAs; as of July 2020), 138 Main-Belt asteroids (MBAs), and 22 comets $^{1}$. More than half have been observed at AO since 2012 after NASA increased their funding of the AO and GSSR radar programs for near-Earth object (NEO) observations. Consequently, the number of observed NEAs at AO increased from less than 30 per year up to 130 per year and tripled for the GSSR radar program. While continued funding at an existing level would double the current total number of radar-characterized NEAs over the next decade, the launch of the Vera C. Rubin Observatory Legacy Survey of Space and Time (LSST) and Near-Earth Object Surveillance Mission (NEOSM) will allow for an expansion of the radar programs by increasing the number of newly discovered NEOs, as well as facilitate robust statistical analyses of the NEO population, and support modeling efforts (see WP Stickle et al.). This white paper focuses on the capabilities of ground-based planetary radar systems to characterize small bodies in the Solar System. For white papers on space-based and hybrid ground- and space-based planetary radar systems and asteroid tomography, i.e., low-frequency radar systems studying the internal 
structure of small bodies, see WP Haynes et al., and for ground-based radar observations of planets and their moons, see WP Rivera-Valentín et al. For a more detailed description about the technical capabilities of the ground-based planetary radar facilities, see Naidu et al. [2].

\section{Sizes, spin states, and shape inversion}

Observations using ground-based radar assets usually provide two types of measurements: Continuous wave (CW) measurements, which provide the Doppler frequency spectrum, and delay-Doppler imaging, which distinguishes the Doppler spectrum at separate ranges. The Doppler echo bandwidth is $B=4 \pi D \cos \delta /(\lambda P)$, where $D$ is the target's diameter, $P$ is its rotation period, $\delta$ is the sub-radar latitude (angle from the target's equator to the closest point to the observer), and $\lambda$ is the wavelength. As evident from the equation, the bandwidth has an ambiguity between the diameter, sub-radar latitude, and the period. If there are no other constraints on the period, for example from light-curve measurements, then the diameter can also be determined based on the delay-Doppler radar images, if the image quality allows. The finest possible range resolution is 7.5 meters when using the Arecibo S-band $(2380 \mathrm{MHz}, \lambda=12.6$ $\mathrm{cm}$ ) radar system of the 305-meter William E. Gordon telescope, 3.75 meters when using the GSSR X-band $(8560 \mathrm{MHz} ; \lambda=3.5 \mathrm{~cm}$ ) radar system on the 70 -meter DSS-14, and 1.875 meters using the GSSR C-band $(7190 \mathrm{MHz}, \lambda=4.2 \mathrm{~cm}$ ) radar system of the 34-meter DSS-13 [2].

Unlike at optical wavelengths, the rotation period of the target plays a major role in the observed signal-to-noise ratio (SNR). While slowly rotating asteroids are easier to observe than fast rotating asteroids due to less Doppler frequency spreading of the echo power, the slowness can cause the echo to be unresolved in frequency. The Doppler resolution is limited by the light round-trip time (RTT) to the target and back and the transmitter-receiver switching time ( $t_{\text {switch }}$ ) so that the finest frequency resolution of the Doppler spectrum is $1 / t_{\text {scan, }}$ where the scan time $t_{\text {scan }}=\mathrm{RTT}-t_{\text {switch. }}$ At AO, the minimum observable RTT is 6 seconds $(900,000 \mathrm{~km})$, and at GSSR 4 seconds. Bistatic observations (receiving the echo with a different radio telescope than that transmitting) can therefore be crucial to better resolve the Doppler frequency when the target is very close or rotates very slowly. A fine example of this were the observations of the km-scale NEA (163899) 2003 SD220 that has a rotation period of 11 days: due to its close proximity to Earth, the monostatic radar images by Arecibo Observatory and GSSR were unresolved, whereas the bistatic radar images of 2003 SD220 using the Green Bank Telescope (GBT) as a receiving antenna were among the most detailed asteroid images obtained using ground-based assets, revealing a ridge and individual boulders on the asteroid's surface [3] (Fig. 1). Radar observations can also be used for detecting non-principal axis rotation (e.g., [4]) and non-gravitational acceleration, i.e., Yarkovsky effect (e.g., [5]) as it changes asteroids' orbits and can be used further to constrain the bulk density and mass of the object, and the YORP effect (e.g., [6]), which changes the rotation rate. This information is a crucial resource to scientific research aligning with the National Near-Earth Object Preparedness Strategy and Action Plan ${ }^{2}$.

Radar observations are the most precise method for NEO astrometry, constrain and complement optical measurements of spin properties, and provide the best size estimates from remote observations, improving our knowledge on the distributions and evolution of those fundamental properties in the NEO population. Continued access to AO, GSSR, and GBT is essential for bistatic as well as monostatic radar opportunities. 
Figure 1. Ground-based radar observations unambiguously reveal binarity and surface features. On the top (from the left): Delay-Doppler images of binary asteroids 2017 YE5 on Jun 26, 2018, by AO, 2016 AZ8 on Jan 4, 2019, by AO, and 2014 HQ124 on Jun 4, 2014, by GSSR DSS-13 and AO (bistatic). The delay (vertical) resolution is 7.5 $\mathrm{m} /$ pixel for 2017 YE5 and 2016 AZ8, and $1.875 \mathrm{~m} /$ pixel for 2014 HQ124. On the bottom: (163899) 2003 SD220 on Dec $15-17,2018$, by GSSR DSS-14 and GBT (bistatic). The delay resolution is $3.75 \mathrm{~m} /$ pixel.

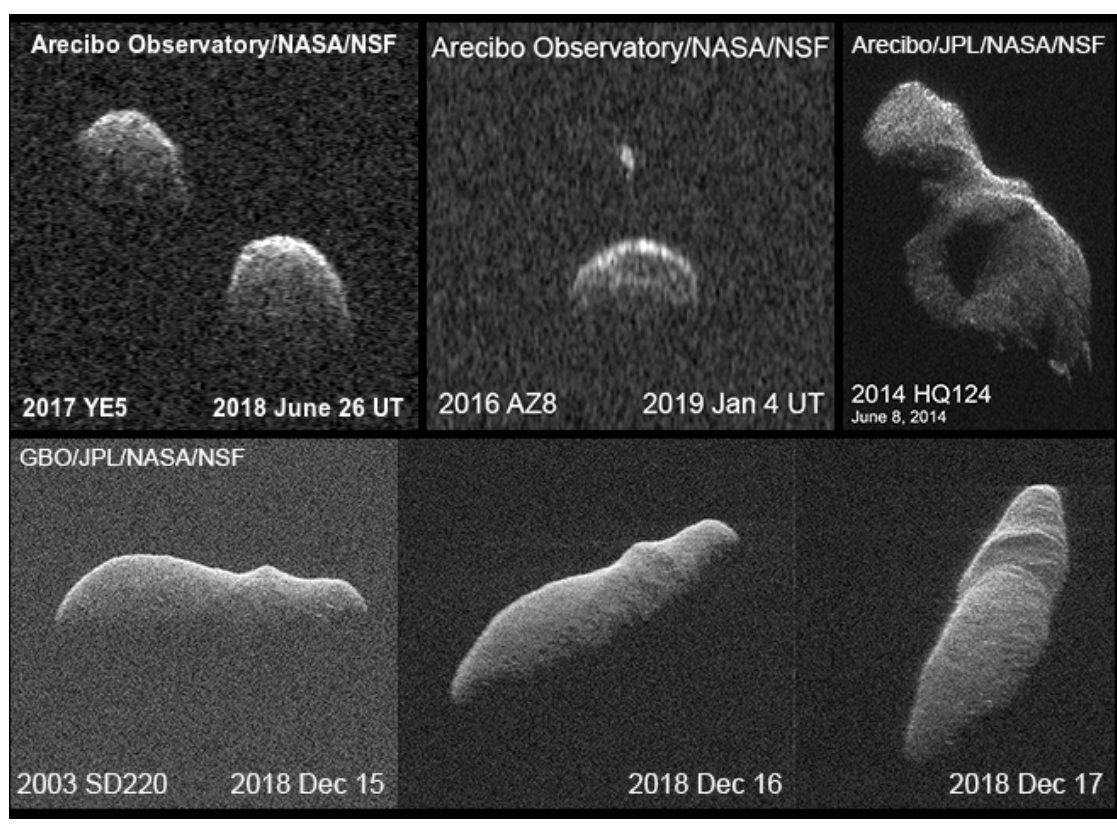

Further, because radar imaging can unambiguously resolve concavities and other features better than other ground-based instruments, they also allow more precise solving of spin pole orientations and shape inversion [7]. Currently, the major challenge in the shape inversion of NEOs based on radar data has been the long computation time needed for spin pole searches and model refining. However, in addition to computers becoming faster, ongoing work based on Bayesian optimization is likely to decrease the spin-pole search time by a factor of three [8]; neural networks have the potential to provide approximate models in seconds [9]; and recent algorithm optimization has been implemented to reduce the model iteration time [10]. Speckle tracking can be used as a further constraint to the spin state and shape. When a radar target is illuminated by a monochromatic CW radar beam, each wavelength-scale patch on its surface re-radiates a portion of the radar echo towards Earth. For rough planetary surfaces, the phases of the echoes from each patch are effectively random. They combine with one another to produce patches of relatively constructive and relatively destructive interference, termed radar speckles. As a radar target spins, the phases of the radar echoes from each patch of surface change uniformly and the speckle pattern moves across Earth $[11,12]$. Radar speckle tracking uses the motion of radar speckles between multiple receive stations to study the target's spin state. Spin state constraints from speckle tracking can enable detailed shape modeling of larger NEAs by breaking the north-south ambiguity present in delay-Doppler images (e.g., [13]) and provide spin state information for very small NEAs where resolved radar imaging is not possible. It can also be used to search for spin state changes due to gravitational tides on asteroids making very close Earth flybys [14], providing information on those objects' moments of inertia. The most common receiving stations for current asteroid speckle tracking observations are elements of the Very Large Baseline Array (VLBA) and GBT, while AO or GSSR transmit. Annually $\sim 5$ targets have SNRs high enough for speckle tracking, providing complementary data to regular radar observations. By the end of the next decade, shape inversion modeling could be effective enough for generating a 
statistically significant sample size of NEO shape and spin-orientation distributions, improving our understanding of the formation and evolution of NEOs. Further research efforts for optimization and implementation of automatized spin-pole-search and shape-inversion methods are needed to reach that goal.

\section{Binaries}

One of the major strengths of radar observations compared to optical observations is directly resolving multibody objects from single-body objects. Binaries can be used for deriving the masses of the components, which constrain the density and internal structure of the asteroids. They play an essential role in our understanding of asteroids' structural and dynamical evolution and are crucial knowledge for spacecraft mission planning to assess the risks to the spacecraft and to model the gravitational field of the target. Approximately 1/6 NEAs are binaries [15]. In total, ground-based radar systems have unambiguously discovered the binary nature of $\sim 70 \%$ of the currently known 64 binary NEAs ${ }^{3}$, including rare equal-mass binaries (e.g., 2017 YE5; see Fig. 1 [16]), and all known ternary NEAs: 3122 Florence, (136617) 1994 CC, (153591) 2001 SN263, and (348400) 2005 JF21. Snapshots of multibody NEAs at different orientations (including whether they are tidally locked) can help us to understand how such systems form and evolve, including non-gravitational effects. Radar observations are a powerful tool for distinguishing binary and ternary NEOs from single bodies and are therefore crucial for understanding their dynamical properties. The comprehensive classification of binary asteroids' size distributions, spin periods, and other physical characteristics such as mass and bulk density estimates provides invaluable information for planetary defense.

\section{Near-Surface Properties Inferred Using Radar Polarimetry}

Ground-based planetary radar systems typically transmit a coherent, coded or unmodulated, circularly polarized signal and receive the echo simultaneously in the same circular (SC) and the opposite circular (OC) polarization as compared to the transmitted signal. The echo power in each polarization can be used to study the surface structure and composition of the target, as the echo power in the SC polarization only arises from multiple scattering or geometrically complex surfaces or wavelength-scale (centimeter-to-decimeter) particles. Traditionally, the ratio of the echo power in the SC polarization to that in the OC polarization - the circular polarization ratio (CPR) - has been used as an approximate gauge of the surface roughness of the target (e.g., [17]). Benner et al. [18] showed that CPR also has different ranges of observed values for different photo-spectrometric classes, demonstrating that the surface roughness depends to some extent on the mineralogical composition. The ongoing OSIRIS-REx mission, though, has demonstrated that the CPR is not always a reliable gauge to the surface roughness. Despite the relatively low CPR observed for 101955 Bennu, which indicated only a moderate number of $\mathrm{cm}$-to-dm-scale particles, the images obtained by the spacecraft revealed a surface covered by a wide size distribution of particles up to multimeter-scale boulders [19]. However, penetration depth adds complexity to the inversion problem because subsurface structure plays a role in addition to the surface reflections, although in some cases the penetration depth of several wavelengths can be considered a unique benefit of radar. Several studies have demonstrated that radar echo especially in the SC polarization (and thus CPR) is enhanced in thick layers of water ice in the permanently shadowed polar craters of Mercury [20] and the 
Galilean moons (e.g., [21]) due to the low absorption properties of water ice at 1-10 GHz. Thick layers of water ice are not necessarily equally relevant to undifferentiated small bodies, but other compositional or morphologic properties are likely to dominate.

While there are many components playing a part in the echo power in each polarization, including the incidence angle and material properties (both structural and chemical) on the near-surface, modeling efforts can help to constrain the range of variables and demonstrate how radar polarimetry can provide information on the effective near-surface bulk density of NEOs [22] with clues to the metal content [23], and in some cases also particle morphology [24]. Also, observations at other wavelengths, or of other targets than NEOs, can provide invaluable insight. For example, several methods originally developed for the lunar surface can support our understanding of NEO surfaces with appropriate adaptation to planetary surfaces with more rubble coverage than lunar surface. For example, the $m$-chi decomposition method [25] can be used for a better identification of regions with different radar scattering properties and thus illustrating the diversity of particle properties on NEO surfaces. Future research efforts are necessary to better understand the role of the $\mathrm{cm}$-to-dm-scale particle properties in the radar signature and at what time scales those properties change. Hybrid analysis of observations with different techniques at different wavelengths is crucial to constrain the large parameter space. Therefore, continued investments in improved analytical and numerical modeling techniques, as well as laboratory measurements over the next decade will provide multi-wavelength polarimetric, photometric, and spectroscopic measurements with crucial support. Especially, research on the connection between spacecraft targets' physical properties and ground-based observations of them are crucial to close the knowledge gaps.

\section{Comets}

Radar is one of the best ground-based observing techniques to directly measure the nucleus of a comet without confusion from the gas and dust comae. In addition, the larger cm-sized coma particles are often detected in the near-nucleus region from their scattering properties, and are easily distinguished from the nucleus (e.g., [26,27]). The CW Doppler spectrum shows the comet nucleus as a strong narrow spike, and the large-grain coma as a skirt extending outward, often in an asymmetric distribution. Particles greater than $2 \mathrm{~cm}$ in diameter dominate the backscattered signal over finer-grained dust and gas. A close approach to Earth is required for radar observations (< $0.25 \mathrm{au}$ ) which is a relatively rare occurrence. In total, only 22 comets have been detected by radar, as compared to close to 1000 NEAs and more than 130 main-belt asteroids. Only eight comet nuclei have been imaged by radar (including two pieces of 73P/Schwassmann-Wachmann 3), and six comet nuclei by spacecraft. Radar images of a comet nucleus reveal the size, spin rate, and surface concavities, from which we can derive a shape model. These images show a wide variety of sizes, shapes, and evolutionary histories; therefore, study of each individual object tells us more about the structure, activity, and possible origin of these enigmatic bodies. Both spacecraft visitations and radar observations have revealed that nuclei seem to have a surprisingly high frequency of contact-binarity. Continued investigation with radar into the shapes of nuclei would improve the statistics and give us confidence on the commonality of contact-binarity. This would have significant implications for understanding cometary evolutionary pathways, especially for Jupiter-family comets originating from an uncertain collisional environment in the Scattered Disk. The cm- 
scale (and larger) grains in the dust coma - among the largest that can be dragged into space by the comet's gas - carry most of the mass that is lost from the comet. The viewing geometry changes from day to day, which provides constraints on the orientation and velocity of the fan of large grains (e.g., [28]). Thus, continued study of the large-grain coma can provide crucial data, in combination with visible and infrared observations, for understanding the overall size distribution of dust leaving the comet. This in turn could be used to better understand the fundamental nature of the activity coming from the nucleus's surface and subsurface layers, as well as the structure of the grains themselves. While none of the currently known comets will have radar apparition opportunities in the next decade, this fact can change as new comets (and interstellar visitors) are discovered.

\section{Small-body mission support}

Planetary radar systems have provided support for a number of planetary missions (WP RiveraValentin et al.) as well as small-body spacecraft missions. Asteroid diameters obtained from radar images support missions such as NEOWISE, Spitzer, Herschel, and upcoming NEOSM (WP Mainzer et al.), which derive asteroid sizes based on optical and infrared measurements using thermal modeling. Comparing the size estimates based on direct imaging by radar helps verifying the reliability of these models. In addition, Arecibo Observatory will support the Double Asteroid Redirection Test (DART) mission in October 2022 by making high-precision astrometric measurements of Dimorphos, 65803 Didymos' satellite, after the planned artificial impact. Radar observations were also the key data for creating the shape model of Didymos [29]. Better shape models improve the reliability of the modeling of the gravitational field of the target object, which mitigates the risks to the spacecraft in the proximity of the target. In addition to the DART mission, radar observations were used for the strikingly precise shape model of the target of the on-going OSIRIS-REx mission, 101955 Bennu [30], for the shape model of 16 Psyche [31], the target of the Psyche mission to be launched in 2022 [32], and the shape model of 3200 Phaethon, the target of the DESTINY+ mission, is a work in progress [33]. Among others ${ }^{4}$, radar observations of asteroid (35107) 1991 VH and (175706) 1996 FG3 will assist the SIMPLEx mission Janus, to be launched in 2022, by valuable ground-based reconnaissance. Another example of an intriguing decadal, if not millennial, NEA observation opportunity in the next decade will be the close-approach of 99942 Apophis in 2029 [34] (also, WP Binzel et al.). The radar apparition in March 2021 will be the next closest approach before 2029 and should provide improved knowledge of its shape, spin state, and possible Yarkovsky effect on its orbit. As described in more detail in the WP Haynes et al., ground-based planetary radar systems could prove to be essential assets for the first radar tomography experiment, i.e., a direct measurement of the internal structure of a NEO, if spacecraft missions utilizing lowfrequency radar receivers or transmitters will be developed for this purpose. Furthermore, targets other than Apophis, especially those smaller NEAs $(<100 \mathrm{~m})$, could be suitable.

\section{Recommendations}

Ground-based planetary radar systems provide a wealth of unparalleled data for planetary science in addition to continuing as a state-of-the-art astrometric tool for planetary defense. Investments on maintaining and upgrading the existing radar facilities with the most recent technologies remains the most cost-effective option for continued access to radar capabilities 
through the next decade and providing the planetary science community with the unique radar capabilities of high-precision astrometry and physical characterization of, e.g., spin state, bulk density, shape, binarity, or decimeter-scale rubble in the near-surface. Multi-wavelength comparisons (radar + optical or different radar wavelengths) can provide necessary constraints to the large parameter space. Therefore, access to a variety of instruments providing data at different wavelengths continues to be important through the next decade. More powerful and effective radar systems than those at Arecibo and Goldstone Observatories have not emerged by 2020 . Further divestment from Arecibo Observatory operations by the National Science Foundation could be detrimental to its planetary radar program. The X-band radar at GSSR, Sband radar at AO, and the planned Ka-band radar at the GBT are complementary, and none can fully replace another. Our key recommendation is that NASA and NSF continue to invest in ground-based radar programs and ensure that the facilities hosting them remain operational and modern, so that the planetary science and planetary defense communities do not lose the unique radar capabilities. We also recommend investing in multi-wavelength analytical and numerical modeling techniques, as well as laboratory development.

\section{References}

[1] Goldstein, R. M. 1969. Science 162, 903.

[2] Naidu, S.P. et al. 2016. AJ 152, 99.

[3] Rivera-Valentin, E.G. et al. 2019. 50th LPSC, abst. id. 3016.

[4] Hudson, R. S., and Ostro, S. J. 1995. Science 270, $5233,84$.

[5] Chesley, S.R. et al. 2003. Science 302, 5651, 1739.

[6] Taylor, P.A. 2007. Science 316, 274.

[7] Magri, C. et al. 2007. Icarus 186, 152.

[8] Marshall, S. et al. 2018. AAS, DPS meeting \#50, id.505.01D.

[9] Busch, M.W. et al. 2019. AGU, Fall Meeting 2019, abstract \#P43E-3513.

[10] Engels, M. et al. 2019. Astronomy and Computing, Volume 28, article id. 100285.

[11] Kholin, I.V., 1988. Radioph. \& Quantum Electr. 31, 371 (transl. from Russian original).

[12] Busch, M.W. et al. 2010. Icarus 209, 535.

[13] Busch, M.W. et al. 2011. Icarus 212, 649.

[14] Reddy, V. et al. 2019. Icarus 326, 133.

[15] Margot, J.L. et al. 2015. “Asteroid Systems: Binaries,...", in Asteroids IV, Univ. of Arizona Press.

[16] Taylor, P.A., et al. 2019. 50th LPSC, abst. id. 2945.

[17] Benner, L.A.M. et al. 2002. MaPS 37, 779.

[18] Benner, L.A.M. et al. 2008. Icarus 198, 294.

[19] Lauretta, D.S. et al. 2019. Nature 568, 7750, 55.

[20] Harmon, J. et al. 2011. Icarus 211, 37.

[21] Ostro, S.J., et al. 1980. Icarus 44, 431.

[22] Magri, C. et al. 2001. MaPS 36, 1697.

[23] Shepard, M.K. et al. 2015. Icarus 245, 38.

[24] Virkki, A.K., and Bhiravarasu, S.S. 2019. JGR:

Planets 124, 11, 3025.

[25] Raney, R. K., et al. 2012. JGR 117, E0OH21.
[26] Harmon, J. et al. 2004. "Radar studies of Comet Nuclei...:", in Comets II. Univ. of Arizona Press.

[27] Virkki, A.K. et al. 2019. Icarus 325, 94.

[28] Harmon, J. et al. 2010. Icarus 207, 499.

[29] Naidu, S.P. et al. 2020. Icarus 348, id. 113777.

[30] Nolan, M.C. et al. 2013. Icarus 226, 629.

[31] Shepard, M.K. et al. 2017. Icarus 281, 388.

[32] Elkins-Tanton, L. et al. 2014. 45th LPSC, abst. id. 1253.

[33] Marshall, S. et al. 2019. EPSC-DPS Joint Meeting 2019, abstract id. EPSC-DPS2019-1246.

[34] Brozovic, M. et al. 2018. Icarus 300, 115.

Cited Decadal White Papers (2020):

Binzel, R.P., et al. "Apophis 2029: Decadal..."

Haynes, M., et al. "Asteroids ... Radar Tomography" Mainzer, A., et al. "Future of Planetary Defense ..." Rivera-Valentín, E.G., et al. “... Ground-Based Radar Observations for Planetary Exploration"

Stickle, A., et al. "The Need for a Well-Defined Modeling Pipeline for Planetary Defense" Taylor, P.A., et al. "Ground-based Radar for Planetary Science and Planetary Defense"

Links:

${ }^{1}$ https://echo.jpl.nasa.gov/asteroids/PDS.asteroid.radar.hi story.html

${ }^{2}$ https://www.nasa.gov/sites/default/files/atoms/files/nati onal near-

earth_object_preparedness_strategy_tagged.pdf

${ }^{3}$ https://echo.jpl.nasa.gov/ lance/binary.neas.html

${ }^{4}$ https://echo.jpl.nasa.gov/ /ance/radar.small.body.missio n.targets.html 